\title{
Prospective Investigation of the Performance of 2 Gamma-Hydroxybutyric Acid Tests: DrugCheck GHB Single Test and Viva-E GHB Immunoassay
}

\author{
Thomas A. Smits, PharmD,* Femke M. J. Gresnigt, MD, $†$ Bianca D. van Groen, PharmD, $\neq$ \\ Eric J. F. Franssen, PharmD, PhD,* and Milly E. Attema-de Jonge, PharmD, PhD $\S$
}

\begin{abstract}
Background: Gamma-hydroxybutyric acid (GHB) is a recreational drug with central nervous system depressing effects that is often abused. A urine GHB point-of-care test can be of great diagnostic value. The objective of this prospective study was to determine the performance of the new DrugCheck GHB Single Test and the Viva-E GHB immunoassay for urine samples in emergency department patients.
\end{abstract}

Methods: Patients presented to the emergency department of the OLVG hospital in Amsterdam with a Glasgow Coma Scale score $<15$ and potential drug of abuse intoxication were included in the study. Between June 2016 and October 2017, 375 patients were included. Using the DrugCheck GHB Single Test (Express Diagnostics Int'l, Blue Earth, MN) and the Viva-E GHB immunoassay (Siemens Healthineers, The Hague, the Netherlands), patients' urine samples were tested for GHB (cutoff for a positive result, 10 or 50 $\mathrm{mcg} / \mathrm{mL}$ GHB). To ensure quality, the results obtained were compared with those generated using a validated gas chromatography method. The tests were considered reliable if specificity and sensitivity were both $>90 \%$. Possible cross-reactivity with ethanol was investigated by analyzing ethanol concentrations in patients' samples.

Results: Seventy percentage of the included patients was men, and the median age was 34 years old. The DrugCheck GHB Single Test's specificity and sensitivity were $90.0 \%$ and $72.9 \%$, respectively, and using $50 \mathrm{mcg} / \mathrm{mL}$ as a cutoff value, its specificity and sensitivity improved to $96.7 \%$ and $75.0 \%$, respectively. Serum and urine ethanol levels in the false-positive group were significantly higher compared with those in the true-negative group. The specificity and sensitivity of the Viva-E GHB immunoassay (cutoff value of $50 \mathrm{mcg} / \mathrm{mL}$ and excluding samples with ethanol levels $\geq 2.0 \mathrm{~g} / \mathrm{L}$ ) were $99.4 \%$ and $93.5 \%$, respectively.

Conclusions: The DrugCheck GHB Single Test's specificity was sufficient, whereas its sensitivity was poor, making it unsuitable for

\section{Received for publication April 10, 2019; accepted June 17, 2019.}

From the *Department of Hospital Pharmacy, OLVG; †Department of Emergency Medicine, OLVG, Amsterdam; †Intensive Care and Department of Pediatric Surgery, Erasmus Medical Centre-Sophia Children's Hospital, Rotterdam; and §Hospital Pharmacy Department, Meander Medisch Centrum, Amersfoort, the Netherlands.

The authors declare no conflicts of interest.

Correspondence: Thomas A. Smits, PharmD, Department of Hospital Pharmacy, OLVG, Oosterpark 9, 1091 AC Amsterdam, the Netherlands (e-mail: T.A.Smits@olvg.nl).

Copyright (c) 2019 Wolters Kluwer Health, Inc. All rights reserved. use at point-of-care. Contrarily, using $50 \mathrm{mcg} / \mathrm{mL}$ as the cutoff value and excluding samples with ethanol levels $\geq 2.0 \mathrm{~g} / \mathrm{L}$, the Viva-E GHB immunoassay showed acceptable results to detect clinically relevant GHB intoxications.

Key Words: GHB, point-of-care, immunoassay, ethanol, emergency department

(Ther Drug Monit 2020;42:139-145)

\section{INTRODUCTION}

Gamma-hydroxybutyric acid (GHB), also known as a rape drug and frequently used in party settings because of its euphoria and libido-increasing effects (chemsex), is a central nervous system depressant drug. Because of its narrow therapeutic range, a small GHB overdose may result in dizziness, sleepiness, hypothermia, or even coma with respiratory depression. ${ }^{1-5}$ In the Netherlands, GHB abuse, which has not changed over the past couple of years, is a significant problem. In 2013 in Amsterdam, 12\% of those who attended rave parties were current users of GHB, and in 2016, $1.3 \%$ of the Dutch population ( $\geq 18$ years) had used GHB at least once. ${ }^{6}$ Moreover, a shift from GHB use to the use of its precursors gamma-butyrolactone and 1,4-butanediol (1,4$\mathrm{BD}$ ), which are rapidly transformed to GHB in vivo and can, therefore, result in intoxication, have recently been described in the literature. ${ }^{5} \mathrm{GHB}$ intoxication is a common cause of impaired consciousness or coma reported at the emergency department (ED) of the OLVG hospital, and each year, about $100 \mathrm{ED}$ visits to this large teaching hospital are GHB-related. Therefore, GHB use is always considered in the differential diagnosis of every patient with a low Glasgow Coma Scale (GCS) score.

To date, it remains difficult to quickly confirm GHB intoxication by rapid GHB detection in urine. Previous studies on GHB analysis focused on enzymatic assays, as well as colorimetric, mass spectrometry, and gas chromatography methods available in laboratories. ${ }^{7-12}$ In some hospitals, the time-consuming gas chromatography with flame ionization detection (GC-FID) method for GHB quantification, which requires highly trained laboratory workers for operation, is available. Obtaining a test result with this method takes about 90 minutes, including sample preparation. However, because of GHB's short half-life (20-40 minutes), ${ }^{7,8,13-15}$ a diagnostic test in ED settings is only helpful if results are quickly available to guide additional 
diagnostic tests and treatment. In the past couple of years, assays for rapid urine GHB detection based on enzymatic methods have become available. Unfortunately, their reliability was inconclusive and showed different results. ${ }^{9,16,17}$ GHB's small nonspecific structural formula makes enzymatic tests prone to cross-reactivity with other similar compounds such as ethanol. ${ }^{9}$ Drogies et $\mathrm{al}^{18}$ reported a novel enzymatic method (Bühlmann Laboratories, Schönenbuch, Switzerland) that was used to measure GHB in serum of 13 patients. The performance of this method was investigated by Hasan et al. Its specificity in serum was $88.9 \%$ (cutoff value, $10 \mathrm{mcg} / \mathrm{mL}$ ), and in urine, it was $98.4 \%$ (cutoff value, $15 \mathrm{mcg} / \mathrm{mL}$ ), indicating a limited possibility of cross-reactivity and leading to a few false-positive results. ${ }^{9}$ Furthermore, small amounts of GHB are endogenously present in humans; therefore, a reliable method should be able to distinguish between physiological concentrations and those resulting from exogenous administration. ${ }^{10,19-21}$ The GHB's cutoff value in the urine needed to confirm exogenous administration is critical for such methods to be reliable. In the literature, urine clinical sample cutoff values of 10 and $15 \mathrm{mcg} / \mathrm{mL}$ have been described because endogenous concentrations in the urine vary between 1 and $10 \mathrm{mcg} / \mathrm{mL}^{10,20-23}$ In this study, the aim was to prospectively investigate the performance of the new DrugCheck GHB Single Test and the Viva-E GHB immunoassay in urine sample analysis in ED patients. The DrugCheck GHB Single Test can be used by ED staff at point-of-care for generating results within 10 minutes, whereas the Viva-E GHB immunoassay, generally used in hospital laboratory settings, is an attractive alternative that can be used to generate results within 15 minutes.

\section{MATERIALS AND METHODS}

\section{Study Design and Setting}

This prospective study was conducted at the ED of the OLVG hospital, a teaching hospital with 2 locations in central Amsterdam (the Netherlands). This ED receives a total of 79,000 patients annually, including many tourists. Intoxications with drugs of abuse are common in the ED of the OLVG hospital, with approximately 1000 intoxications each year. Ethical approval for this study was obtained from the hospital's institutional review board, and the Medical Research Ethics Committees United (MEC-U) waived the need for a formal ethics approval according to the Dutch law on medical research in humans.

\section{Subjects}

All patients presented at the ED of the OLVG hospital between June 2016 and October 2017, with a differential diagnosis including drugs of abuse intoxication, were included in the study if their GCS score was below 15 . In addition, patients with a GCS score of 15 were also included if GHB intoxication was suspected. Given that patients were presented to the ED at different times, each visit was counted as a new case. Urine and blood samples (in a $\mathrm{B}-\mathrm{NaF} / \mathrm{KOx}$ tube) were collected by a nurse in compliance with routine care, stored in a refrigerator $\left(2-8^{\circ} \mathrm{C}\right)$ for 24 hours, and subsequently transferred into a freezer $\left(-20^{\circ} \mathrm{C}\right)$ within $1-3$ days (See "Methods of measurement" for GHB stability). All measurements were performed using residual specimen that was available after routine care use. Patients were included if urine was obtained.

\section{Methods of Measurement GHB On-Site Test}

Urine samples were used to examine the performance of the on-site DrugCheck GHB Test (Express Diagnostics Int'l, Blue Earth, MN). This was further named as the "GHB on-site test", which is based on the reaction between GHB and nicotinamide adenine dinucleotide (NAD), producing $\mathrm{NADH}+$ (catalyzed by GHB dehydrogenase). If GHB was present, a diaphorase couple tetrazolium dye reaction will change the color of the dipstick to purple. The GHB on-site test dipstick was dipped into the urine sample for 5 seconds and examined after 2 minutes (or after 10 minutes, if it was not purple after 2 minutes). ${ }^{11,24}$ If urine GHB concentration was higher than $10 \mathrm{mcg} / \mathrm{mL}$, the result was considered positive for GHB. ${ }^{20,21}$ Results were stratified into 2 categories: negative/endogenous (colorless, $0-10 \mathrm{mcg} / \mathrm{mL}$ ) and positive/ exogenous (light purple, $10-50 \mathrm{mcg} / \mathrm{mL}$; dark purple, $>50$ $\mathrm{mcg} / \mathrm{mL}$ ) (Fig. 1). According to the manufacturer, vitamin $\mathrm{C}$ (ascorbic acid) interferes with the GHB on-site test and was therefore checked with an additional dipstick in case of a positive GHB test. ${ }^{24}$ When its concentration appeared to be higher than $10 \mathrm{mg} / \mathrm{dL}$, the GHB on-site test result was considered unreliable, and such samples were not included in the GHB on-site test's performance analysis. In this study, the GHB onsite test was performed by pharmaceutical laboratory workers at the hospital pharmacy. Results were not shared with the doctors, ensuring their treatment plan was not changed based on the results of a nonvalidated test.

\section{The Viva-E GHB Immunoassay}

Given that the GHB on-site test could produce unreliable results, we tested an alternative fast GHB quantification method: the Viva-E GHB immunoassay (Siemens Healthineers, the Hague, the Netherlands), in combination with a GHB enzymatic assay kit (Bühlmann Laboratories,

\section{GHB Color Chart}

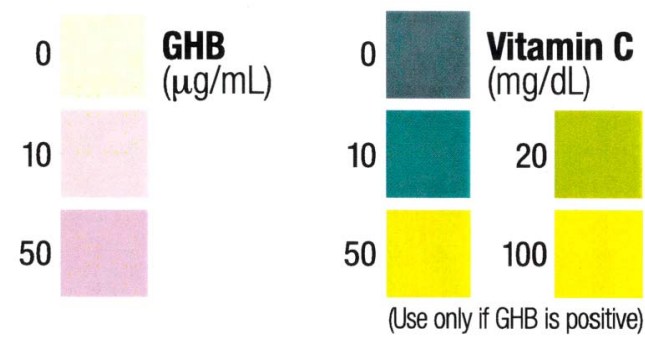

DC532A 0815

FIGURE 1. Reference colors to quantify urine GHB and vitamin C using the DrugCheck GHB Single Test. ${ }^{24}$ 
Schönenbuch, Switzerland). This assay was also based on GHB-induced NADH+ production, where a shift in absorbance was correlated with the GHB concentration. ${ }^{25}$ It is a laboratory-based assay that produces sample measurements within 15 minutes. All urine samples examined with the GHB on-site test were also tested using the Viva-E GHB immunoassay.

\section{Gold Standard}

Results of the GHB on-site test and Viva-E GHB immunoassay were compared with those from the gold standard method: a validated GC-FID system. A full validation based on the "Guidelines on Bioanalytical Method Validation" of the European Medicine Agency was successfully completed before this study. ${ }^{26}$ The GC-FID system consisted of an HP 6890 Series G1530A Gas Chromatograph, a G2613A/G2614A automatic liquid sampler, and a microcell electron capture detector (Agilent Technologies, Amstelveen, the Netherlands). The column was a Chrompack, WCOT-fused silica capillary column (CP-SIL $5 \mathrm{CB}, 25 \mathrm{~m}$ $\times 530 \mu \mathrm{m}$, film thickness $500 \mu \mathrm{m}$; Agilent Technologies), at a temperature of $120^{\circ} \mathrm{C}$. $\mathrm{y}$-Valerolactone was used as the internal standard. The method was based on the conversion of GHB into gamma-butyrolactone in an acid environment. ${ }^{20}$

\section{GHB Stability in Urine}

All measurements were performed within 28 days of sampling. GHB stability in urine was confirmed in-house within the 28 days while stored in the refrigerator $\left(2-8^{\circ} \mathrm{C}\right)$ or freezer $\left(-20^{\circ} \mathrm{C}\right)$, for both pooled and synthetic urine at 2 different concentrations ( 10 and $200 \mathrm{mcg} / \mathrm{mL}$ ). The recovery after 28 days was between $95 \%$ and $106 \%$ in all cases and was therefore accepted.

\section{Interference of Ethanol}

To examine for possible ethanol interference with test results, ethanol concentrations were measured in urine and serum samples using the same GC-FID system and column, at a temperature of $90^{\circ} \mathrm{C}$. Ethanol concentrations were compared between the true-negative and false-positive groups, to investigate if ethanol could induce false positives in GHB negative samples. Serum ethanol concentrations were measured, given that this specimen is often used for measuring ethanol concentrations in clinical practice. We also investigated the ethanol concentration in urine because urine ethanol had the ability to interfere with the tests. Owing to the possible ethanol interference, the specificity and sensitivity were equally calculated, excluding samples with a serum ethanol concentration $\geq 2.0 \mathrm{~g} / \mathrm{L}$. This cutoff value was used because these ethanol concentrations are known to increase GHB levels. ${ }^{25}$

\section{False-Positive Results}

The GHB on-site test's false positives were subject to an additional screening in serum to identify possible interfering substances. The screening method consisted of a multistage mass spectrometer: LC-MS ${ }^{\mathrm{n}}$ (LC-MS ${ }^{\mathrm{n}}$ AmaZon Speed ToxTyper; Bruker, Bremen, Germany) system, including an ultra-high-performance liquid chromatography system
(Dionex ultimate 3000 RS pump, autosampler and column compartment; Thermo Scientific, Breda, the Netherlands). The column used was an Acclaim Rapid Separation LC column $(120 \mathrm{C} 18,2,1 \times 100 \mathrm{~mm}$, film thickness $2.2 \mu \mathrm{m}, 120 \mathrm{~A}$; Thermo Scientific) at a temperature of $40^{\circ} \mathrm{C}$. Samples were prepared using a precipitation method with acetonitrile. An extensive library (Toxtyper 1.1 Library, May 2014) with 900 drugs (including drugs of abuse) and metabolites was used to identify possible interfering substances. ${ }^{27}$

\section{Outcomes}

The primary outcomes were the specificity and sensitivity of the GHB on-site test and the Viva-E GHB immunoassay. Specificity and sensitivity were calculated as follows:

1. Specificity $=$ number of true negatives/(number of true negatives + number of false-positive samples).

2. Sensitivity $=$ number of true positives/(number of true positives + number of false-negative samples).

The specificity and sensitivity were calculated for the regular cutoff value of $10 \mathrm{mcg} / \mathrm{mL}$ and a higher value, 50 $\mathrm{mcg} / \mathrm{mL}$. According to the OLVG hospital, most patients with clinically relevant GHB intoxications have $>50 \mathrm{mcg} / \mathrm{mL}$ GHB levels. This cutoff has not been described in the existing literature, but we propose that it can be used to detect clinically relevant GHB intoxications. However, the $50 \mathrm{mcg} / \mathrm{mL}$ cutoff is too high to draw conclusions on patient use of GHB because urine levels $>10 \mathrm{mcg} / \mathrm{mL}$ are already associated with GHB intake. ${ }^{10,20-23}$

Secondary outcomes included the difference in ethanol levels between the false-positive and true-negative groups and possible interfering substances in the false-positive group.

\section{Analyses}

The GHB on-site test and the Viva-E GHB immunoassay were considered reliable if their specificity and sensitivity were both $>90 \%$. To calculate the sample size, a 0.95 proportion with a 2 -sided confidence interval of $95 \%$ and a total width of $0.10(0.90-1.00)$ was used. The ClopperPearson formula was used to calculate the $95 \%$ confidence interval. A sample size of 94 patients with a positive GHB test and 94 patients with a negative GHB test was required. The sample size calculation was performed using PASS 14 (NCSS LLC, Kaysville, UT). IBM SPSS Statistics (version 22; IBM, Armonk, NY) was used for statistical analyses. Only the Mann-Whitney $U$ test was used for non-normally distributed continuous data. $P$ values $<0.05$ were considered statistically significant.

\section{RESULTS}

\section{Study Subjects}

Of 375 patients included in the study, 103 tested positive for urine GHB following the gold standard and a cutoff value of $10 \mathrm{mcg} / \mathrm{mL}$. Patient characteristics are displayed in Table 1 . Most enrolled subjects were men: $81.6 \%$ in the GHB-positive and $66.2 \%$ in the GHB-negative group. In the GHB-positive and -negative groups, mainly 
TABLE 1. Patient Characteristics

\begin{tabular}{lcc}
\hline & $\begin{array}{c}\text { GHB-Positive } \\
(\mathbf{n}=\mathbf{1 0 3})\end{array}$ & $\begin{array}{c}\text { GHB-Negative } \\
(\mathbf{n}=\mathbf{2 7 2})\end{array}$ \\
\hline Age, median (IQR, 25-75) & $34(28-39)$ & $34(23-47)$ \\
Male, no. (\%) & $84(81.6)$ & $180(66.2)$ \\
Residential country, no. (\%) & & \\
$\quad$ The Netherlands & $94(91)$ & $194(71)$ \\
Europe (other) & $4(4)$ & $56(21)$ \\
$\quad$ Other/unknown & $5(5)$ & $22(8)$ \\
GCS, median (IQR, 25-75) & $3(3-8)$ & $10(3-14)$ \\
\hline
\end{tabular}

Dutch residents were observed ( $91 \%$ and $71 \%$, respectively). The GCS score was significantly lower in the GHB-positive group than in the GHB-negative group $(P<0.001)$. Serum samples were obtained from 175 patients.

\section{Specificity and Sensitivity}

Based on the results of the GHB on-site test on the 375 obtained urine samples, a total of 121 samples tested positive and 254 samples negative. Of the GHB-positive patients, 44 were also positive for vitamin C. The GHB on-site test's results were therefore unreliable for these patients (following product information) and, hence, were excluded. Of the 44 excluded patients, 33 tested positive and 11 tested negative for GHB based on the gold standard method.

The GHB on-site test's results of the 331 patients left were used to calculate the specificity and sensitivity for 2 cutoff values, and the results are shown in Tables 2 and 3. The specificity and sensitivity values for the GHB on-site test were $90.0 \%$ and $72.9 \%$, respectively (cutoff $10 \mathrm{mcg} / \mathrm{mL}$ ).

More so, the Viva-E GHB immunoassay's results for all patients were compared with the gold standard. The specificity and sensitivity values of the Viva-E GHB immunoassay are shown in Table 3 . The specificity and sensitivity values for the Viva-E GHB immunoassay were $93.6 \%$ and $85.0 \%$, respectively (cutoff, $10 \mathrm{mcg} / \mathrm{mL}$ ).

\section{Ethanol}

Ethanol interference with the GHB on-site test and Viva-E GHB immunoassay was investigated in all samples. The urine ethanol concentrations of the true-negative and the false-positive groups are displayed in Figure 2 (cutoff, 10 $\mathrm{mcg} / \mathrm{mL}$ ). More extensive data on urine and serum ethanol concentrations are shown in Table 4. Ethanol concentrations were significantly higher in the false-positive group than the true-negative group (cutoff, $10 \mathrm{mcg} / \mathrm{mL})(P<0.001)$. Owing

TABLE 2. Correlation Between the GHB On-Site Test and the Gold Standard GC-FID System

\begin{tabular}{lccr}
\hline & \multicolumn{3}{c}{$\begin{array}{c}\text { GC-FID System (Gold Standard) } \\
\text { (Cutoff, 10 mcg/mL) }\end{array}$} \\
\cline { 2 - 4 } GHB On-Site Test & Positive & Negative & Total \\
\hline Positive & 51 & 26 & 77 \\
Negative & 19 & 235 & 254 \\
Total & 70 & 261 & 331 \\
\hline
\end{tabular}

to the possible interference of ethanol, the specificity and sensitivity were calculated using a cutoff value of $50 \mathrm{mcg} /$ $\mathrm{mL}$, excluding samples (41) with a serum ethanol concentration $\geq 2.0 \mathrm{~g} / \mathrm{L}$. None of the 41 excluded samples was positive for GHB (gold standard). For these results, the specificity and sensitivity of the GHB on-site test were $100 \%$ and $72.7 \%$, respectively, whereas those for the Viva-E GHB immunoassay were $99.4 \%$ and $93.5 \%$, respectively (Table 3 ).

\section{Vitamin C}

To investigate the actual interference of vitamin $\mathrm{C}$, the specificity and sensitivity of the GHB on-site test were also calculated, without excluding vitamin C-positive samples. Results are shown in Table 3. The specificity decreased from $90.0 \%$ to $86.4 \%$ when GHB results (cutoff $10 \mathrm{mcg} / \mathrm{mL}$ ) of positive vitamin $\mathrm{C}$ samples were included in the analyses. The sensitivity increased from $72.9 \%$ to $81.6 \%$.

\section{False Positives}

False-positive samples of the GHB on-site test were qualitatively screened for possible interfering substances in the serum. Serum samples were available in 15 of the 26 false-positive samples. Detected substances (apart from ethanol) were highly heterogeneous, containing, for instance, venlafaxine, baclofen, ethyl glucuronide, midazolam, theobromine, MDMA, MDA, hydrocortisone, cocaine, and benzoylecgonine. No specific substance was identified as prominent in multiple false-positive samples.

\section{DISCUSSION}

To the best of our knowledge, this is the first prospective study regarding tests for urine GHB, which can be quickly assessed in the hospital. The major findings of this study were the poor performance of the GHB on-site test and the possibility of the Viva-E GHB immunoassay being an acceptable alternative for detecting clinically relevant GHB intoxications (cutoff, $50 \mathrm{mcg} / \mathrm{mL}$ ). By contrast, the Viva-E GHB immunoassay registered poor performance at a cutoff value of $10 \mathrm{mcg} / \mathrm{mL}$ and is therefore unable to detect exogenous GHB administration in cases of low urinary concentrations. ${ }^{10,20-23}$ Compared with the gas chromatography method used as the gold standard in this study, the main advantage of the investigated methods is the ability to obtain quicker test results. An on-site test for detecting urine GHB within minutes can be of great diagnostic value in addition to point-of-care tests for other drugs of abuse. ${ }^{28-35}$ However, the goal of achieving a specificity and sensitivity of $>90 \%$ was unmet. The GHB on-site test's specificity was at the borderline $(90.0 \%)$, and its sensitivity was unacceptable $(72.9 \%)$. Actually, the sensitivity is even worse due to the 33 GHBpositive patients who were excluded because of a positive vitamin $\mathrm{C}$ test. Moreover, the large number of positive vitamin $C$ tests $(44 / 121 ; 36 \%)$ makes this test inapplicable for clinical practice. Vitamin $\mathrm{C}$ could have caused a false-positive result in 11 of the 44 positive vitamin $\mathrm{C}$ samples $(25 \%)$, which is a substantial percentage. Including the positive vitamin C samples resulted in increased sensitivity $(81.6 \%)$ and 
TABLE 3. The Specificity and Sensitivity of the GHB On-Site Test and the Viva-E GHB Immunoassay Calculated With 2 Different Cutoffs (10 and $50 \mathrm{mcg} / \mathrm{mL}$ )

\begin{tabular}{|c|c|c|c|c|c|c|}
\hline & Positive Vitamin C Samples & Samples EtOH $\geq 2.0 \mathrm{~g} / \mathrm{L}$ & Specificity, \% & Sensitivity, \% & Specificity, \% & Sensitivity, \% \\
\hline & Included in Analyses & Included in Analyses & Cutoff, & $\mathrm{mcg} / \mathrm{mL}$ & Cutoff, & $\mathrm{mcg} / \mathrm{mL}$ \\
\hline \multirow[t]{2}{*}{ GHB on-site test } & No & Yes & 90.0 & 72.9 & 96.7 & 75.0 \\
\hline & No & No & 97.5 & 83.3 & 100 & 72.7 \\
\hline \multirow{2}{*}{$\begin{array}{l}\text { Viva-E GHB } \\
\text { immunoassay }\end{array}$} & - & Yes & 93.6 & 85.0 & 99.6 & 88.3 \\
\hline & - & No & 99.1 & 74.1 & 99.4 & 93.5 \\
\hline
\end{tabular}

EtOH, ethanol.

decreased specificity $(<90 \%)$. Irrespective of these, the GHB on-site test is inapplicable for clinical practice.

The Viva-E GHB immunoassay showed better results, with an acceptable specificity of $93.6 \%$, albeit with an insufficient sensitivity of $85.0 \%$. This specificity is comparable with the specificity calculated by Hasan et al (98.4\%) using the same assay, but a different cutoff value $(15 \mathrm{mcg} /$ $\mathrm{mL}$ ). The sensitivity Hasan et $\mathrm{al}^{9}$ found was better: $100 \%$, but this was based on a small sample size $(n=36)$ and could therefore not be fairly compared with our data. The specificity of both tests was higher when using a higher cutoff value of $50 \mathrm{mcg} / \mathrm{mL}$ (GHB on-site test: $96.7 \%$ and Viva-E GHB immunoassay: $99.6 \%$ ), resulting in less false positives. This higher cutoff value leads to a higher specificity because interfering agents may have less influence when the test turns positive at a higher GHB concentration. The sensitivity of both tests also increased when using the higher cutoff value of $50 \mathrm{mcg} / \mathrm{mL}$ (GHB on-site test: $75.0 \%$ and Viva-E GHB immunoassay: $88.3 \%$ ). The slight increase in sensitivity was unexpected but could be explained by the decrease in falsenegative samples at the cutoff of $50 \mathrm{mcg} / \mathrm{mL}$. Specifically, 6 samples had GHB concentrations between 10 and $50 \mathrm{mcg} / \mathrm{mL}$ (gold standard), of which 5 and 4 samples tested negative $(<10 \mathrm{mcg} / \mathrm{mL})$ for the on-site GHB test and the Viva-E GHB immunoassay, respectively. These false-negative results were eliminated at the cutoff of $50 \mathrm{mcg} / \mathrm{mL}$. Both tests had substantial false-negative results (sensitivity $<90 \%$ ), indicating that the tests encounter difficulties in detecting GHB.

Nevertheless, the cutoff value of $50 \mathrm{mcg} / \mathrm{mL}$ has an important drawback because it could only indicate clinically relevant GHB intoxications. A negative result with this cutoff value could not rule out the fact that the patient suffered from exogenous GHB administration. The results of the Viva-E GHB immunoassay should therefore only be used to confirm a suspicion of GHB intake, and no conclusions should be drawn upon obtaining negative results. Additional measurements with a chromatographic method are still necessary for demonstrating exogenous GHB administration.

Given that the number of generated false-negative results was not acceptable for the GHB on-site test, regardless of the cutoff value used, the test cannot be safely used to identify patients who have used GHB. A negative test result may lead to further diagnostic work-up and adequate patient diagnosis and treatment. A positive test result may support physicians in their clinical decision-making and possibly prevent unnecessary diagnostics. However, false-negative results may lead to redundant diagnostics and suboptimal treatment, and even worse, false-positive results may result in an increased health risk for patients. More specifically, it may withhold the physician from applying further diagnostic tools, potentially missing another cause of a decreased GCS score. Hence, because of the impaired sensitivity of the test, we do not recommend using the GHB on-site test in clinical practice.

Our data support the hypothesis that false-positive results are caused by the concurrent presence of ethanol in the urine. The specificity of both tests $(>90 \%)$ was acceptable and the ethanol influence tolerable. By contrast, the sensitivity was unexpectedly low, without any concrete explanations. We postulate that unknown interfering substances present in the samples probably obstruct or delay enzymatic activity during the conversion of NAD into NADH+. Conversely, the specificity and sensitivity of the Viva-E GHB immunoassay were good, at a cutoff value of $50 \mathrm{mcg} / \mathrm{mL}$ and exclusion of samples with a serum ethanol concentration $\geq 2.0 \mathrm{~g} / \mathrm{L}$ ( $99.4 \%$ and $93.5 \%$, respectively). Concomitant use of ethanol and GHB occurs regularly, especially in drug-facilitated sexual assault where GHB is added to alcoholic beverages. ${ }^{36}$ In this population, high ethanol concentrations in combination with GHB cause toxic effects. Although the specificity of the test is sufficient $(>90 \%)$, it is necessary to confirm the absence or presence of GHB with a validated chromatography method in case of possible legal matters.

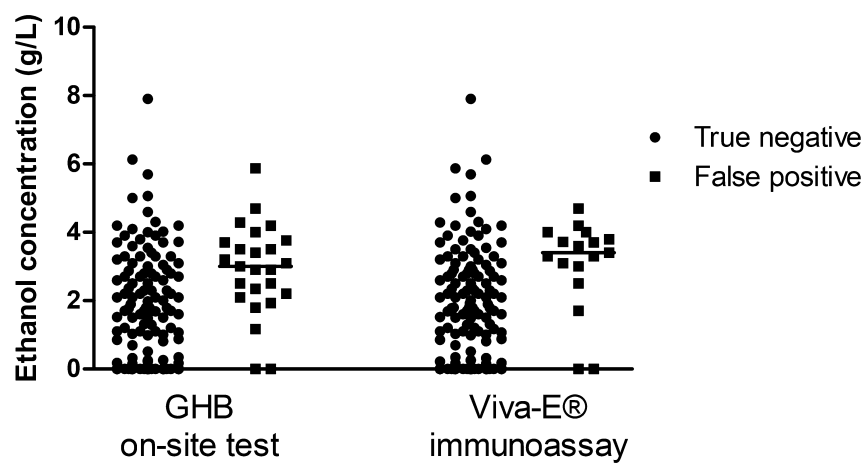

FIGURE 2. Urine ethanol concentrations for the true-negative and false-positive groups (cutoff, $10 \mathrm{mcg} / \mathrm{mL}$ ) of the $\mathrm{GHB}$ onsite test and the Viva-E GHB immunoassay. 
TABLE 4. Ethanol Concentrations of the True-Negative and False-Positive Groups for the GHB On-Site Test (Positive Vitamin C Samples Were Excluded) and Viva-E Immunoassay Using a Cutoff Value of $10 \mathrm{mcg} / \mathrm{mL}$

\begin{tabular}{|c|c|c|c|}
\hline $\begin{array}{l}\text { Ethanol Levels (Median, 25-75 } \\
\text { Percentile, g/L) }\end{array}$ & True-Negative & False-Positive & $\boldsymbol{P}$ \\
\hline GHB on-site test, urine & $0.0(0.0-1.8)(\mathrm{n}=232)$ & $3.0(2.2-3.7)(\mathrm{n}=25)$ & $<0.001$ \\
\hline GHB on-site test, serum & $0.0(0.0-1.9)(\mathrm{n}=100)$ & $2.5(2.2-3.1)(\mathrm{n}=13)$ & $<0.001$ \\
\hline Viva-E GHB immunoassay, urine & $0.0(0.0-2.0)(\mathrm{n}=245)$ & $3.4(2.8-3.9)(\mathrm{n}=17)$ & $<0.001$ \\
\hline Viva-E GHB immunoassay, serum & $0.0(0.0-2.1)(\mathrm{n}=106)$ & $2.8(2.2-3.4)(\mathrm{n}=9)$ & $<0.001$ \\
\hline
\end{tabular}

This study covers the analytical validation of the GHB on-site test and the Viva-E GHB immunoassay. Given that our expected outcomes were unmet, we conclude that further improvements of these commercially available on-site tests are necessary for obtaining a specificity and sensitivity $>90 \%$. This study also emphasizes the importance of validating all novel on-site and laboratory tests before implementation in clinical practice. More so, before implementing a test in clinical practice at the ED, the clinical relevance should be studied by investigating whether a quickly available GHB level contributes to patient outcome.

A limitation of this study is that the GHB on-site test was performed by laboratory workers of the pharmaceutical laboratory in the hospital pharmacy. Ideally, after implementation in clinical practice, the GHB on-site test is used by ED nurses or physicians for fast results at the point-ofcare, although laboratory workers are specifically trained to perform different types of analyses and to follow standard operating procedures. The sometimes-hectic ED is a suboptimal place to perform an analytical test. Critical steps in the analytical procedure of the GHB on-site test are reading the results after a given time, performing an additional test for vitamin $\mathrm{C}$, and comparing the color of the dipstick with reference colors. The specificity and sensitivity of the test may, therefore, be different in a real-life setting, where the test is operated by nurses or physicians at the point-of-care. However, the analytical validation is preferably performed by laboratory workers to assess the performance of the test itself, without external interfering factors. Hence, this study was suitable to study the performance of the GHB on-site test.

Second, the required sample size of 94 positive patients was not achieved for the GHB on-site test because of sample exclusion (vitamin C-positive samples). ${ }^{19}$ This has implications for the reliability of the calculated specificity and sensitivity of the GHB on-site test. However, the large number of positive vitamin $\mathrm{C}$ results in this study population makes this test unusable in clinical practice. Moreover, the sample size was sufficient to determine the specificity and sensitivity for the Viva-E GHB immunoassay, where vitamin $\mathrm{C}$ measurements and eventual exclusions were not required.

Finally, our sample GHB concentrations were not considered very challenging for the test. Only 13 of the 375 tested samples had a GHB concentration between 5 and 100 $\mathrm{mcg} / \mathrm{mL}$ (gold standard), within the range of the cutoff values. The other samples were either negative or clearly positive $(>100 \mathrm{mcg} / \mathrm{mL})$. To challenge these tests, it would have been better to have more samples around the cutoff values of 10 and $50 \mathrm{mcg} / \mathrm{mL}$. On the other hand, real patient samples were used in this study, reflecting GHB levels seen in clinical practice.

\section{CONCLUSIONS}

In summary, the DrugCheck GHB on-site test showed a specificity and sensitivity of $90.0 \%$ and $72.9 \%$, respectively. Therefore, we do not recommend this GHB on-site test be implemented at the ED. The Viva-E GHB immunoassay would be an acceptable alternative to detect clinically relevant GHB intoxications using a cutoff of $50 \mathrm{mcg} / \mathrm{mL}$, provided patients with a serum ethanol concentration $\geq 2.0 \mathrm{~g} / \mathrm{L}$ are excluded. Under these conditions, the specificity and sensitivity of the Viva-E GHB immunoassay were $99.4 \%$ and $93.5 \%$, respectively.

\section{ACKNOWLEDGMENTS}

The authors acknowledge the physicians and nurses of the ED department of the OLVG hospital (Amsterdam, the Netherlands) for their contribution to patient sample collection. They also acknowledge the OLVG hospital's pharmacy laboratory workers (Martijn Brancart, Ralph Brands, Jolanda Dijk-van Kuilenburg, Stefan Dommisse, and Sanne Neijzing) for their excellent analytical work, without which this study could not have been accomplished.

\section{REFERENCES}

1. Wong CGT, Chan KFY, Gibson KM, et al. $\gamma$-Hydroxybutyric acid: neurobiology and toxicology of a recreational drug. Toxicol Rev. 2004; 23:3-20.

2. Chin RL, Sporer KA, Cullison B, et al. Clinical course of $\gamma$-hydroxybutyrate overdose. Ann Emerg Med. 1998;31:716-722.

3. Li J, Stokes SA, Woeckener A. A tale of novel intoxication: a review of the effects of $\gamma$-hydroxybutyric acid with recommendations for management. Ann Emerg Med. 1998;31:729-736.

4. Schep LJ, Knudsen K, Slaughter RJ, et al. The clinical toxicology of gamma-hydroxybutyrate, gamma-butyrolactone and 1,4-butanediol. Clin Toxicol (Phila). 2012;50:458-470.

5. Busardò FP, Gottardi M, Tini A, et al. Replacing GHB with GBL in recreational settings: a new trend in chemsex. Curr Drug Metab. 2018; 19:1080-1085.

6. Van Laar MW, van Gestel B, Cruts AAN, et al. National Drug Monitor 2017. Utrecht, the Netherlands: Trimbos Institute; 2018. Available at: https://www. trimbos.n1/kerncijfers/nationale-drug-monitor. Accessed December 11, 2018.

7. Palatini P, Tedeschi L, Frison G, et al. Dose-dependent absorption and elimination of gamma-hydroxybutyric acid in healthy volunteers. Eur $J$ Clin Pharmacol. 1993;45:353-356.

8. Brenneisen R, ElSohly MA, Murphy TP, et al. Pharmacokinetics and excretion of gamma-hydroxybutyrate (GHB) in healthy subjects. J Anal Toxicol. 2004;28:625-630. 
9. Hasan L, Jermann TM, Weber JM, et al. An enzymatic method to determine $\gamma$-hydroxybutyric acid in serum and urine. Ther Drug Monit. 2011; 33:757-765.

10. Kang S, Oh SM, Chung KH, et al. A surrogate analyte-based LC-MS/MS method for the determination of $\gamma$-hydroxybutyrate (GHB) in human urine and variation of endogenous urinary concentrations of GHB. $J$ Pharm Biomed Anal. 2014;98:193-200.

11. Bravo DT, Harris DO, Parsons SM. Reliable, sensitive, rapid and quantitative enzyme-based assay for gamma-hydroxybutyric acid (GHB). $J$ Forensic Sci. 2004;49:379-387.

12. Alston WC, $\mathrm{Ng} \mathrm{K}$. Rapid colorimetric screening test for $\gamma$-hydroxybutyric acid (liquid X) in humane urine. Forensic Sci Int. 2002;126:114 117.

13. Ferrara SD, Zotti S, Tedeschi L, et al. Pharmacokinetics of $\gamma$-hydroxybutyric acid in alcohol dependent patients after single and repeated oral doses. Br J Clin Pharmacol. 1992;34:231-235.

14. Borgen LA, Okerholm R, Morrison D, et al. The influence of gender and food on the pharmacokinetics of sodium oxybate oral solution in healthy subjects. J Clin Pharmacol. 2003;43:59-65.

15. Abanades S, Farré M, Segura M, et al. $\gamma$-Hydroxybutyrate (GHB) in humans. Pharmacodynamics and pharmacokinetics. Ann N Y Acad Sci. 2006;1074:559-576.

16. Sciotti MA, Hasan L, Scholer A, et al. Development and characterization of an enzymatic method for the rapid determination of gamma hydroxybutyric acid. Chimia (Aarau). 2010;64:793-798.

17. Andresen H, Aydin BE, Mueller A, et al. An overview of gammahydroxybutyric acid: pharmacodynamics, pharmacokinetics, toxic effects, addiction, analytical methods, and interpretation of results. Drug Test Anal. 2011;3:560-568.

18. Drogies T, Willenberg A, Ramshorn-Zimmer A, et al. Detection of gamma hydroxybutyrate in emergency department: nice to have or a valuable diagnostic tool? Hum Exp Toxicol. 2016;35:785-792.

19. Bessman SP, Fishbein WN. Gamma-hydroxybutyrate, a normal brain metabolite. Nature. 1963;200:1207-1208.

20. LeBeau MA, Montgomery MA, Miller ML, et al. Analysis of biofluids for gamma-hydroxybutyrate $(\mathrm{GHB})$ and gamma-butyrolactone (GBL) by headspace GC-FID and GC-MS. J Anal Toxicol. 2000;24: 421-428.

21. Kankaanpää A, Liukkonen R, Ariniemi K. Determination of $\gamma$-hydroxybutyrate (GHB) and its precursors in blood and urine samples: a saltingout approach. Forensic Sci Int. 2007;170:133-138.
22. Busardò FP, Jones AW. Interpreting $\gamma$-hydroxybutyrate concentrations for clinical and forensic purposes. Clin Toxicol (Phila). 2019;57:149163 .

23. Busardò FP, Kyriakou C. GHB in biological specimens: which cut-off levels should be taken into consideration in forensic toxicological investigation? Recent Pat Biotechnol. 2014;8:206-214.

24. DrugCheck. Product Insert: GHB (Gamma-hydroxybutyric Acid). Accessed December 11, 2018.

25. Product Information GHB Gamma-hydroxybutyric acid Enzymatic Assay on Clinical Chemistry Analyzers. Schönenbuch/Basel, Switzerland: Bühlmann Laboratories AG. Accessed December 11, 2018.

26. European Medicines Agency. Guideline on Bioanalytical Method Validation. 2011. Available at: https://www.ema.europa.eu/en/documents/ scientific-guideline/guideline-bioanalytical-method-validation_en.pdf. Accessed May 2, 2016.

27. Bruker Daltonik GmbH. Toxtyper TM 1.1 Libraries Reference Manual Revision 2 (May 2014). Accessed December 11, 2018.

28. Lager PS, Attema-de Jonge ME, Gorzeman MP, et al. Clinical value of drugs of abuse point of care testing in an emergency department setting. Toxicol Rep. 2017;5:12-17.

29. Attema-de Jonge ME, Peeters SYG, Franssen EJF. Performance of three point-of-care urinalysis test devices for drugs of abuse and therapeutic drugs applied in the emergency department. J Emerg Med. 2012;42:682691.

30. Hammett-Stabler CA, Pesce AJ, Cannon DJ. Urine drug screening in the medical setting. Clin Chim Acta. 2002;315:125-135.

31. Yang JM. Toxicology and drugs of abuse testing at the point of care. Clin Lab Med. 2001;21:363-374.

32. George S, Braithwaite RA. Use of on-site testing for drugs of abuse. Clin Chem. 2002;48:1639-1646.

33. Crouch DJ, Hersch RK, Cook RF, et al. A field evaluation of five on-site drug-testing devices. J Anal Toxicol. 2002;26:493-499.

34. Peace MR, Poklis JL, Tarnai LD, et al. An evaluation of the OnTrak Testcup-er on-site urine drug-testing device for drugs commonly encountered from emergency departments. J Anal Toxicol. 2002;26:500-503.

35. Peace MR, Tarnai LD, Poklis A. Performance evaluation of four on-site drug-testing devices for detection of drugs of abuse in urine. J Anal Toxicol. 2000;24:589-594.

36. Grela A, Gautam L, Cole MD. A multifactorial critical appraisal of substances found in drug facilitated sexual assault cases. Forensic Sci Int. 2018;292:50-60. 